\title{
Upregulation of SOX9 inhibits the growth of human and mouse melanomas and restores their sensitivity to retinoic acid
}

\author{
Thierry Passeron, Julio C. Valencia, Takeshi Namiki, Wilfred D. Vieira, Hélène Passeron, \\ Yoshinori Miyamura, and Vincent J. Hearing
}

Laboratory of Cell Biology, National Cancer Institute, NIH, Bethesda, Maryland, USA.

\begin{abstract}
Treatments for primary and metastatic melanomas are rarely effective. Even therapeutics such as retinoic acid (RA) that are successfully used to treat several other forms of cancer are ineffective. Recent evidence indicates that the antiproliferative effects of RA are mediated by the transcription factor SOX 9 in human cancer cell lines. As we have previously shown that SOX9 is expressed in normal melanocytes, here we investigated SOX9 expression and function in human melanomas. Although SOX9 was expressed in normal human skin, it was increasingly downregulated as melanocytes progressed to the premalignant and then the malignant and metastatic states. Overexpression of SOX9 in both human and mouse melanoma cell lines induced cell cycle arrest by increasing p21 transcription and restored sensitivity to RA by downregulating expression of PRAME, a melanoma antigen. Furthermore, SOX9 overexpression in melanoma cell lines inhibited tumorigenicity both in mice and in a human ex vivo model of melanoma. Treatment of melanoma cell lines with PGD2 increased SOX9 expression and restored sensitivity to RA. Thus, combined treatment with PGD2 and RA substantially decreased tumor growth in human ex vivo and mouse in vivo models of melanoma. The results of our experiments targeting SOX9 provide insight into the pathophysiology of melanoma. Further, the effects of SOX9 on melanoma cell proliferation and RA sensitivity suggest the encouraging possibility of a noncytotoxic approach to the treatment of melanoma.
\end{abstract}

\section{Introduction}

Melanomas are generally resistant to radiotherapy, and even under the best circumstances, chemotherapy can usually only provide a few additional months of survival. Although used with success in several cancers, such as leukemia and some breast cancers, retinoic acid (RA) or its derivatives are ineffective in treating patients with melanoma. PRAME is a melanoma antigen expressed in melanomas and many cancer cell lines. PRAME acts as a dominant repressor of the RA receptor, and recent evidence indicates that silencing of PRAME restores sensitivity to RA $(1,2)$. Although little is known about the mechanism(s) controlling PRAME expression, downregulation of that protein by tumor cells would be expected to play a critical role in melanoma therapy.

SOX9 is a transcription factor that plays a key role in sex determination and chondrogenesis during development (3, 4). Several recent studies have demonstrated that SOX9 plays active roles in adult tissues as well (5-7). Recently, we reported that SOX9 directly activates the microphthalmia transcription factor (MITF) promoter (8), which is critical for regulating the differentiation of normal melanocytes and also for modulating the proliferation of melanoma cells (9). Interestingly, recent evidence suggests that the antiproliferative effects of SOX9 are mediated by RA in human cancer cell lines (10). Thus, the key interactions of SOX9 with

Conflict of interest: Thierry Passeron and Vincent J. Hearing are listed as coinventors on a patent filed by the National Cancer Institute for the use of SOX9 to target melanomas.

Nonstandard abbreviations used: MITF, microphthalmia transcription factor; RA, retinoic acid.

Citation for this article: J. Clin. Invest. 119:954-963 (2009). doi:10.1172/JCI34015. melanoma survival factors and RA make it an emerging prime target for melanoma therapy.

For those reasons, we have characterized the mechanism by which SOX9 exerts its antiproliferative effects on melanoma cells and tumors. We report that SOX9 expression was downregulated in 37 of 39 melanoma specimens examined. Analysis of an additional set of specimens revealed that SOX9 was expressed in only $18 \%$ of nevi, in less than $4 \%$ of primary melanomas, and in none of the metastatic melanoma specimens tested. We show that SOX9 functions by binding the $\mathrm{p} 21$ promoter, which results in a strong suppression of cell growth, and further that SOX9 decreases the expression of PRAME and restores the sensitivity of melanoma cells to RA. In fact, stronger antiproliferative effects of RA were obtained after cells were stably transfected with SOX9. Interestingly, treatment with PGD2 upregulated the expression of endogenous SOX9, which in turn downregulated PRAME and restored sensitivity to RA. We found, using mouse and reconstructed human skin models, that overexpression of SOX9 prevented melanoma cell invasion and metastatic spread. Finally, we show that pharmacological treatment with BW245C, which activates the PGD2 pathway, combined with RA treatment decreased the growth of melanoma tumors in mouse and in human ex vivo melanoma models. Therefore, upregulation of SOX9 expression in human melanomas, which has the potential to dramatically slow the growth of tumors and enhance their sensitivity to RA treatment, represents a novel approach for melanoma therapy.

\section{Results}

SOX9 expression is downregulated in nevi and in melanomas. We analyzed the expression patterns of SOX9 using a tissue array containing 39 

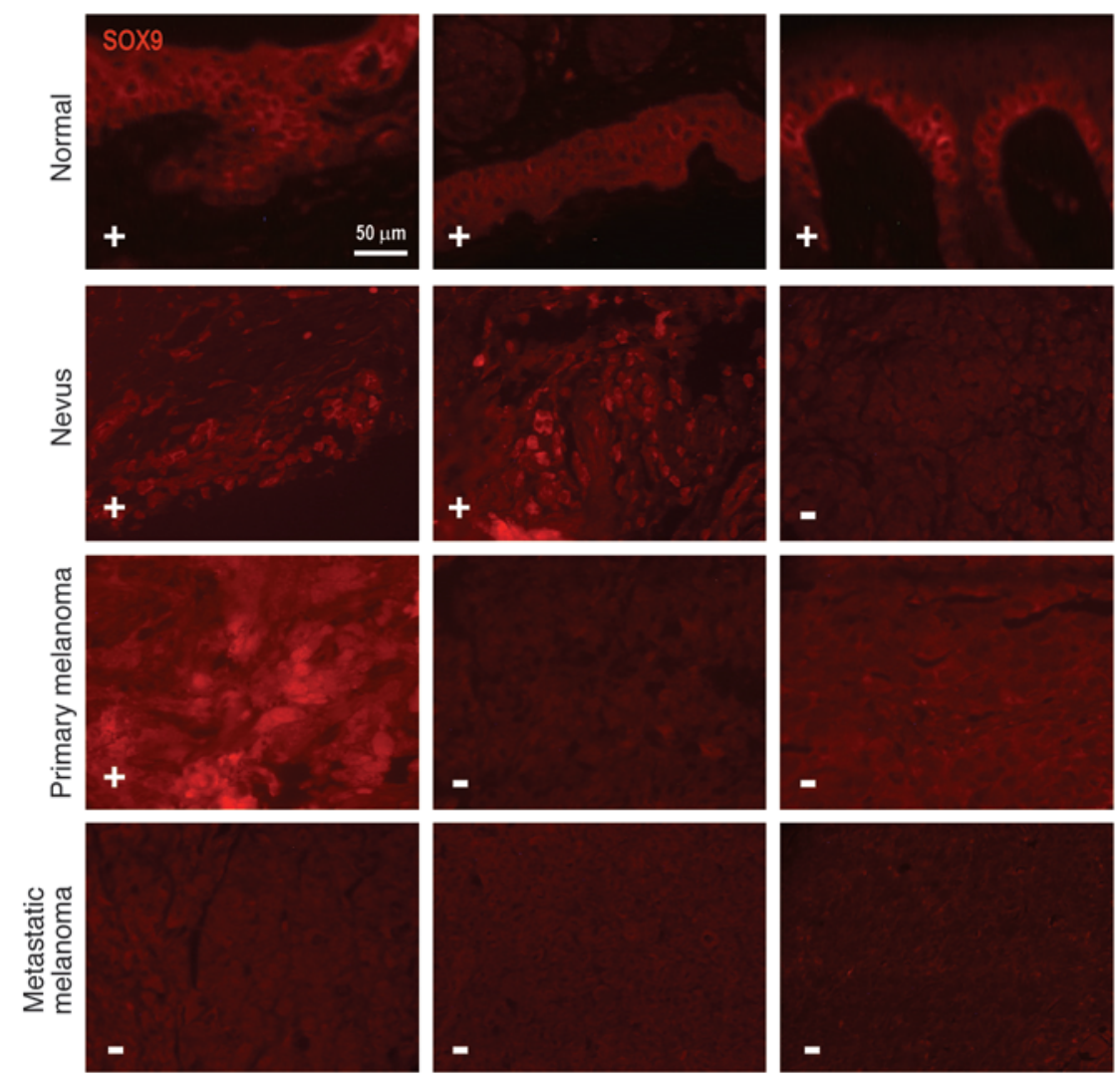
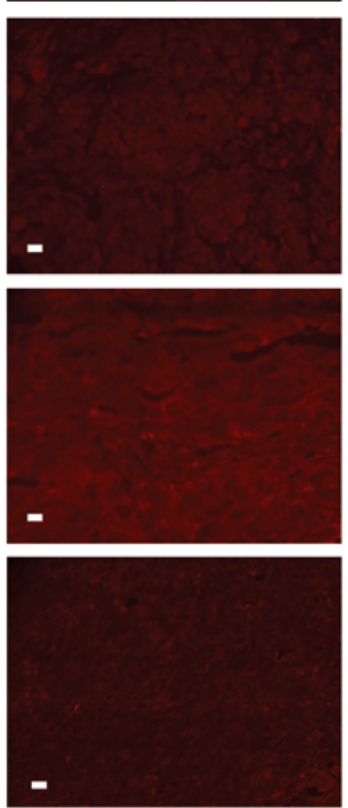

\section{Figure 1}

Expression of SOX9 in normal skin, nevi, and melanomas in vivo. Immunohistochemistry of paraffin-embedded sections of the ME1001 human melanoma tissue array stained with the SOX9 antibody (red). Three representative specimens from each group are shown (results summarized in Table 1). +, positive staining; - , negative staining. Scale bar: $50 \mu \mathrm{m}$. human melanoma specimens. Immunohistochemistry confirmed that SOX9 expression was weak or negative in 37 (95\%) of the 39 melanoma specimens (Supplemental Figure 1; supplemental material available online with this article; doi:10.1172/JCI34015DS1). Similarly, we studied the relative expression of SOX9 in normal skin exposed or unexposed to UVB (Supplemental Figure 1). In agreement with our previous results (8), SOX9 was expressed in normal human melanocytes (positive for the melanocytic marker MART1), and its expression increased dramatically after UVB exposure. To confirm these data and to further study the expression of SOX9 in vivo, we used immunohistochemistry of a different human tissue array to stain 22 nevi (and surrounding perilesional skin), 56 primary melanomas, and 20 metastatic melanomas. The expression of SOX9 was positive in the normal skin areas, but weak or negative in $81.8 \%$ (18 of 22 ) of nevi, in $96.4 \%$ (54 of 56) of primary melanomas, and in 100\% (20 of 20) of metastatic melanomas (Figure 1 and Table 1).Thus, SOX9 expression decreases as melanocytic cells progress from the normal condition to the premalignant (nevi) to the transformed state and is completely negative in the most advanced (metastatic) state of malignancy.

SOX 9 induces cell cycle arrest by upregulating $p 21$ and MITF expression in melanoma cells. MITF controls the proliferation of melanoma cells through the cyclin-dependent kinase inhibitor p21 $1^{\mathrm{CIP} 1}$ (hereafter called p21) (9). In addition, SOX9 also regulates pigmentation through direct binding to MITF in melanocytes (8). Based on the marked downregulation of SOX9 expression observed in primary and in metastatic melanomas, we hypothesized that SOX9 may have a role in regulating the proliferation of melanocytic cells. To test that hypothesis, we transiently transfected either a SOX9 cDNA or an empty cDNA vector into 3 human melanoma cell lines
(A375, Mel Juso, and SK-Mel28) and into 1 murine melanoma cell line (B16/F10). After 15 days, all 4 melanoma cell lines transfected with SOX9 showed a significant decrease in proliferation $(P<0.05)$ compared with controls (Figure 2, A-C). To determine whether the reduced proliferation was due to cell cycle arrest or to an increase in apoptosis, we analyzed the cell cycle profile of human A375 melanoma cells and murine B16/F10 melanoma cells, stably transfected with SOX9 or with a vector expressing only GFP. Interestingly, the cell cycle profile confirmed that cells overexpressing SOX9 showed marked cell cycle arrest in the $G_{1}$ phase, demonstrating that the decrease in proliferation is caused by cell cycle arrest (Figure 2D). To determine whether the SOX9-induced $\mathrm{G}_{1}$ arrest required $\mathrm{p} 21$ activation, we overexpressed SOX9 in A375 cells containing a BRAF mutation (A375-SOX9) or in Mel Juso cells containing a NRAS mutation. These mutations are known to downregulate the antiproliferative effects of MITF (11). Immunoblot analysis confirmed

\section{Table 1}

Expression of SOX9 in nevi, primary melanomas, and metastatic melanomas in vivo

\begin{tabular}{lccc} 
Specimen & SoX9- & S0X9+ & Total \\
Nevi & $81.8 \%(n=18)$ & $18.2 \%(n=4)$ & 22 \\
Primary melanoma & $96.4 \%(n=54)$ & $3.6 \%(n=2)$ & 56 \\
Metastatic melanoma & $100 \%(n=20)$ & $0 \%(n=0)$ & 20 \\
\hline
\end{tabular}

Results of immunohistochemistry on paraffin-embedded sections of the ME1001 human melanoma tissue array stained with the SOX9 antibody. The percentage of tumor specimens positive and negative for SOX9 is reported for nevi, primary melanomas, and metastatic melanomas. 
A
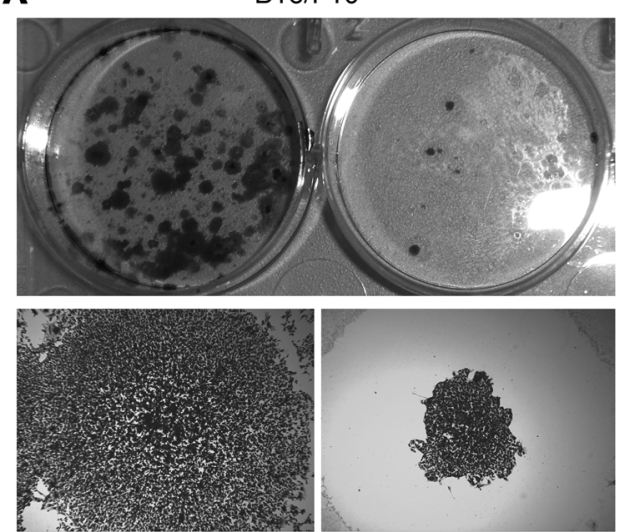

Control

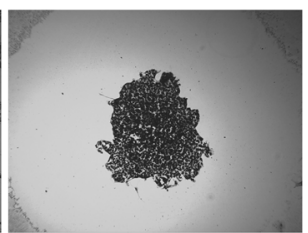

SOX9

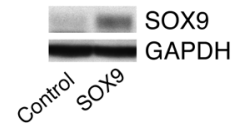

B

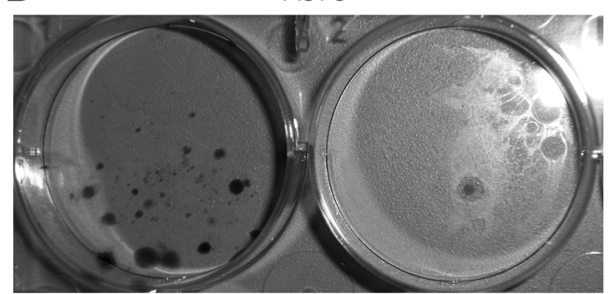

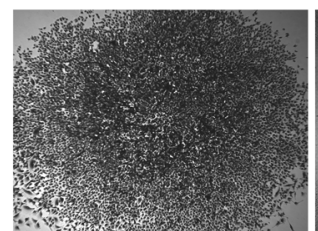

Control

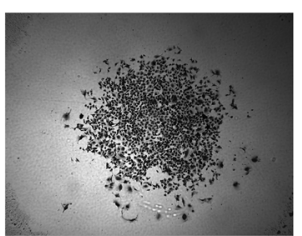

sox9

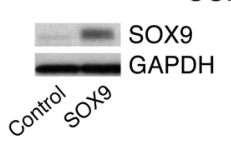

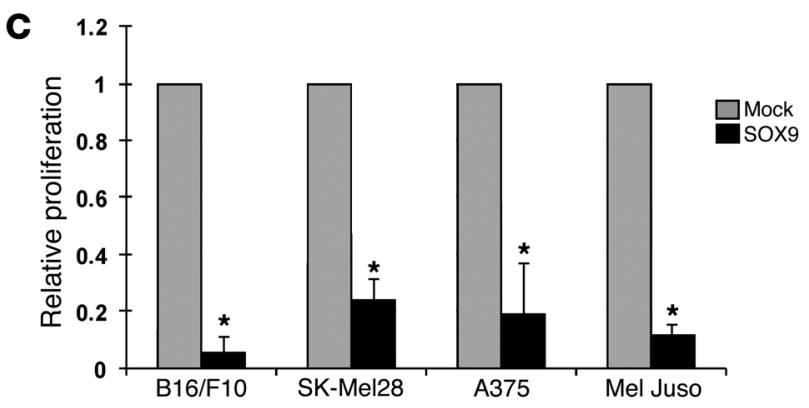

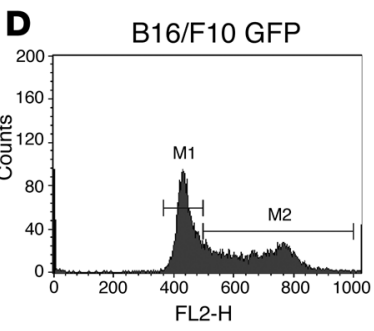

$\mathrm{G}_{1}=48.90 \%$

$G_{1} /\left(S+G_{2}\right)=1$

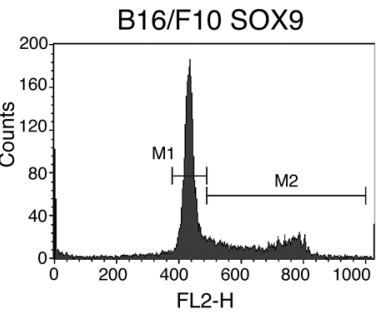

$\mathrm{G}_{1}=61.09 \%$

$G_{1} /\left(S+G_{2}\right)=1.9$

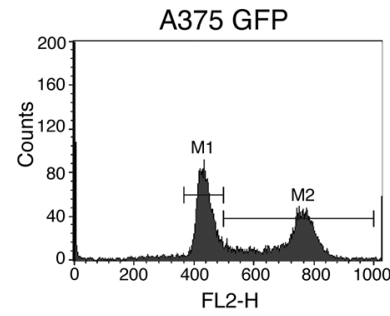

$\mathrm{G}_{1}=44.2 \%$

$G_{1} /\left(S+G_{2}\right)=0.94$

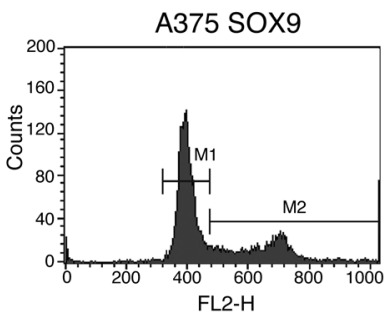

$\mathrm{G}_{1}=67.5 \%$

$G_{1} /\left(S+G_{2}\right)=2.15$

Figure 2

SOX9 decreases the proliferation of melanoma cells. (A) B16/F10 murine melanoma cells were cotransfected with a puromycin resistance vector and either an empty vector (Control) or the SOX9 CDNA (SOX9) and were grown in medium containing puromycin for 15 days. Upper panel: Macroscopic view of the proliferative clones. Middle panels: The same clones at a magnification of $\times 10$. An immunoblot of proteins extracted from those cells using antibodies against SOX9 and GAPDH is shown. (B) A375 human melanoma cells were cotransfected with a puromycin resistance vector and with either an empty vector or the SOX9 cDNA and were grown in medium containing puromycin for 15 days. Upper panel: Macroscopic view of the proliferative clones. Middle panels: The same clones at a magnification of $\times 10$. An immunoblot of proteins extracted from those cells using antibodies against SOX9 and GAPDH is shown. (C) Relative proliferation of SOX9-transfected cells compared with the control for B16/F10, SK-Mel28, A375, and Mel Juso melanoma cell lines. ${ }^{*} P<0.001$. (D) SOX9 induces melanoma cell arrest in the $G_{1}$ phase. B16/F10 and A375 melanoma cells were transduced with SOX9 lentivirus or with GFP lentivirus as a control and then were selected with blasticidin. After 5 days, the cells were stained with propidium iodide, and the cell cycle was studied using FACS analysis. Percentages of cells in $G_{1}$ are indicated. $G_{1} /\left(S+G_{2}\right)$, ratio of cells in $G_{1}$ to cells in $S$ plus $G_{2}$.

that the expression of $\mathrm{p} 21$ was increased by the overexpression of SOX9 in both cell lines (Figure 3A). Interestingly, SOX9 markedly increased MITF levels in A375 cells, but the increase in MITF was very slight in Mel Juso melanoma cells. This latter observation was difficult to reconcile with the strong increase in p21 expression also observed in the Mel Juso cells (see below). Immunofluorescence confirmed that both p21 and MITF signals were increased in A375 cells overexpressing SOX9 (Figure 3B). Similar results were obtained in Mel Juso and B16/F10 melanoma cells (data not shown). These results are consistent with findings from our previ- 
A

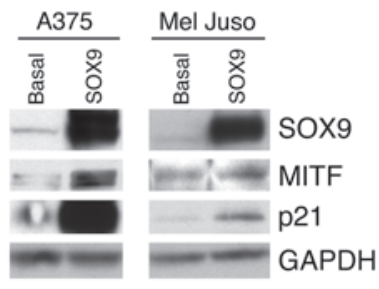

B
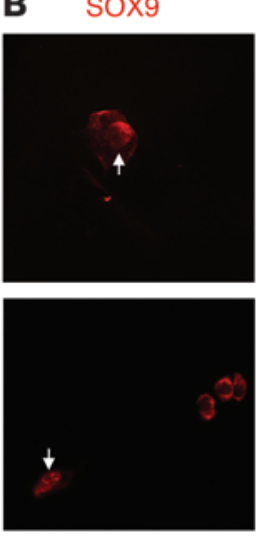
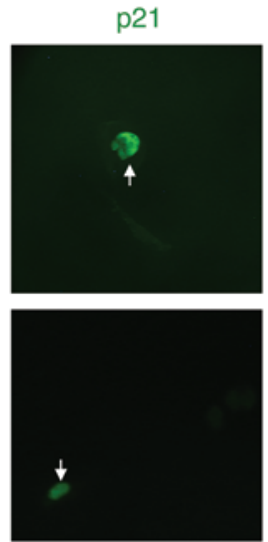

D

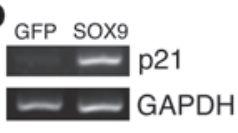

GAPDH
E

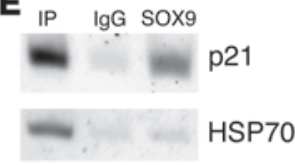

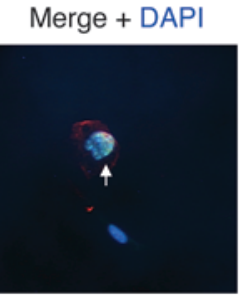

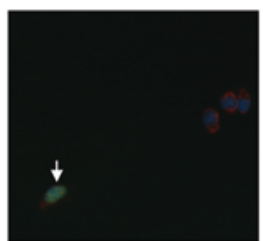

SOX9
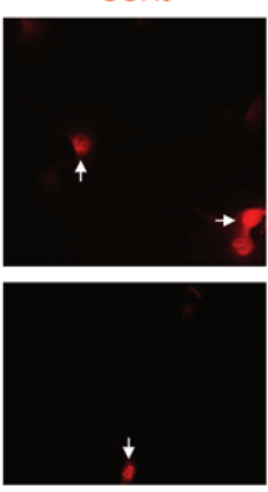

MITF
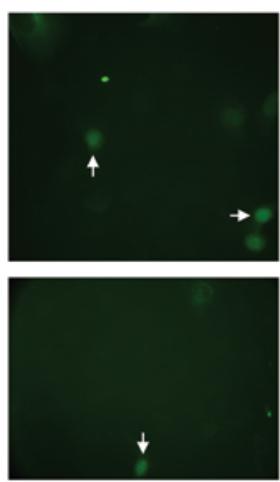

Merge + DAPI
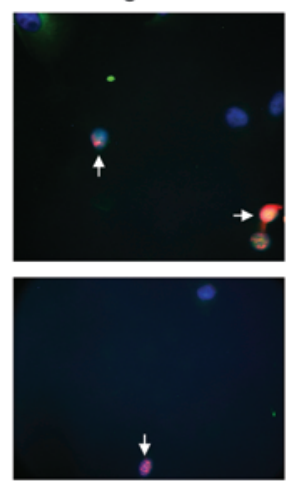

C

Control
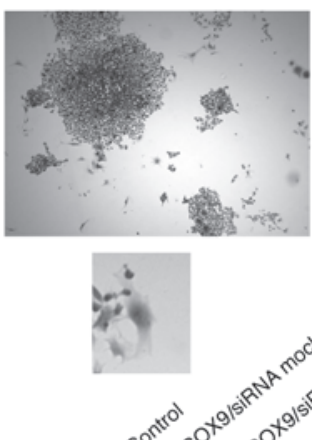

SOX9/siRNA mock
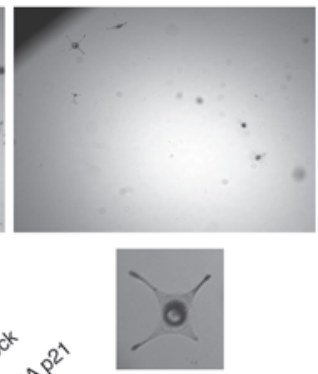

SOX9/siRNA p21

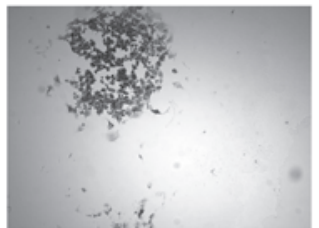

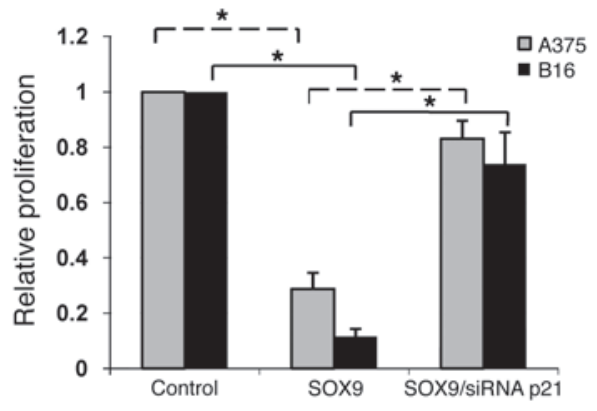

p21

SOX9

GAPDH

Figure 3

SOX9 acts on MITF and p21 to inhibit proliferation. (A) A375 and Mel Juso melanoma cells were transduced with SOX9 lentivirus or with GFP lentivirus as a control. One week after selection with blasticidin, proteins were extracted and analyzed using immunoblotting with SOX9, p21, MITF, and GAPDH antibodies. (B) A375 melanoma cells were transfected with SOX9 cDNA and were fixed 48 hours after transfection and stained for SOX9 (red), DAPI (blue), and either p21 (left panels) or MITF (right panels) antibodies (green). Transfected cells are identified with arrows. Original magnification, $\times 20$. (C) Proliferation assay of B16/F10 murine melanoma cells comparing empty vector (Control); SOX9 cDNA combined with siRNA mock (SOX9/siRNA mock); and SOX9 cDNA combined with siRNA of p21 (SOX9/siRNA p21). Assays were stopped after 10 days, and images are shown at a magnification of $\times 10$ (upper panels). The lower panels show higher-magnification $(\times 40)$ images of individual cells. Note the clear differentiation observed in SOX9-transfected cells with melanosomes accumulating at the peripheries of the dendrites. The graph shows the relative proliferation of SOX9-transfected B16 or A375 cells with or without silencing of p21 compared with the controls $\left({ }^{\star} P<0.001\right)$. The immunoblot below shows proteins extracted from B16/F10 cells in the same conditions using antibodies against SOX9, p21, and GAPDH. (D) Expression levels of the p21 using RT-PCR in A375 cells transfected for 48 hours with GFP or cDNA SOX9 cDNA. GAPDH expression served as the control. (E) ChIP assays performed on A375 melanoma cells using IgG or SOX9 antibodies. Immunoprecipitated DNAs were analyzed using PCR with p21 and HSP70 primers.

ous studies on normal human melanocytes (8). To further confirm the results, we knocked down 21 expression using an siRNA or a mock siRNA in A375 and in B16/F10 cells overexpressing SOX9. Immunoblot analysis confirmed the complete reduction of p21 protein levels in those cells (Figure 3C). The siRNA-mediated inhi- bition of $\mathrm{p} 21$ also allowed a significant $(P<0.05)$ rescue of colony formation activity in A375-SOX9 and in B16-SOX9 cells compared with controls (Figure 3C). RT-PCR analysis of A375 cells overexpressing SOX9 showed that the increase in $\mathrm{p} 21$ protein expression was due to increased transcription of the p21 gene (Figure 3D). 

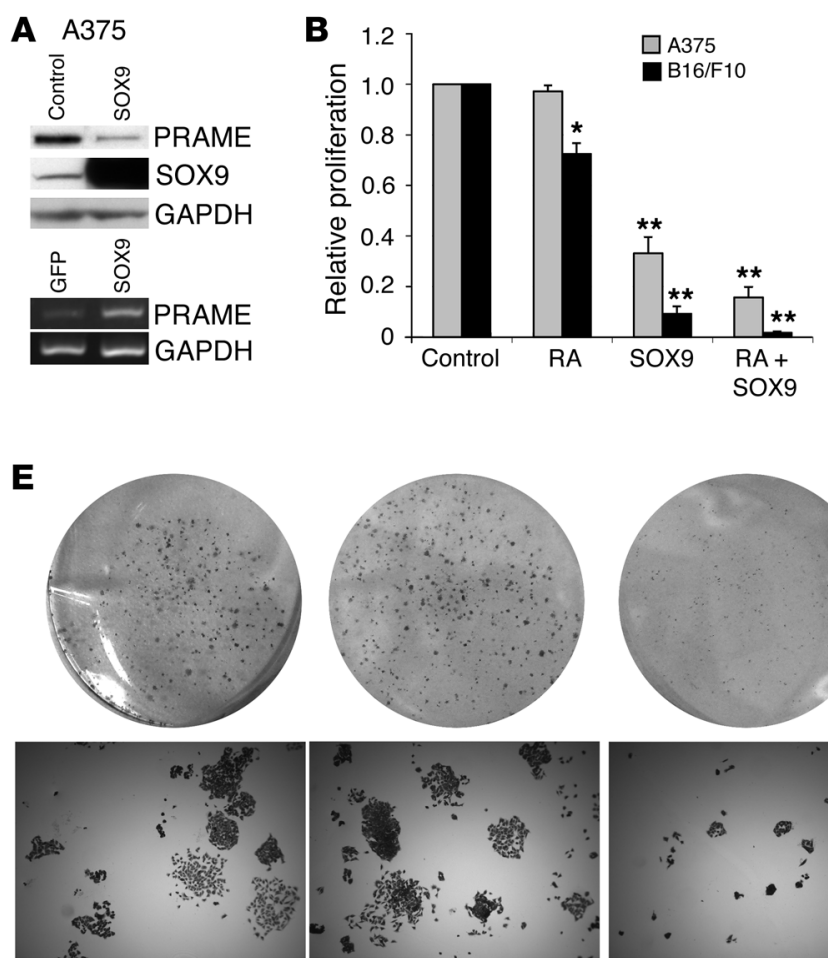

Basal
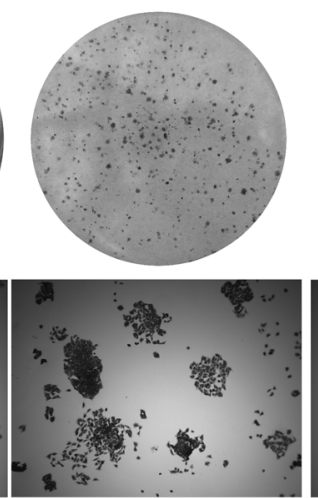

RA
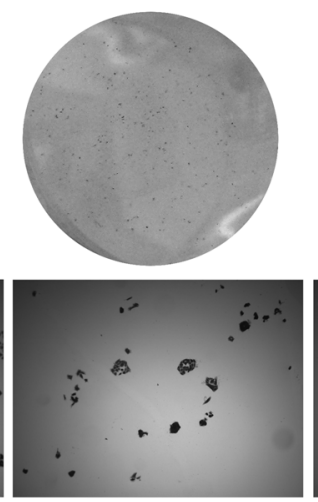

PGD2
C

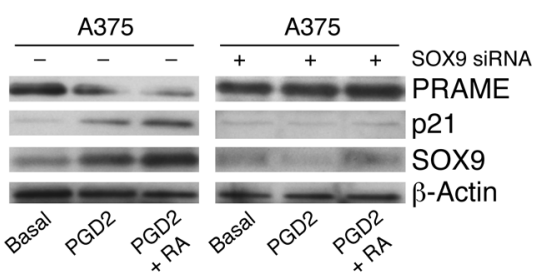

D
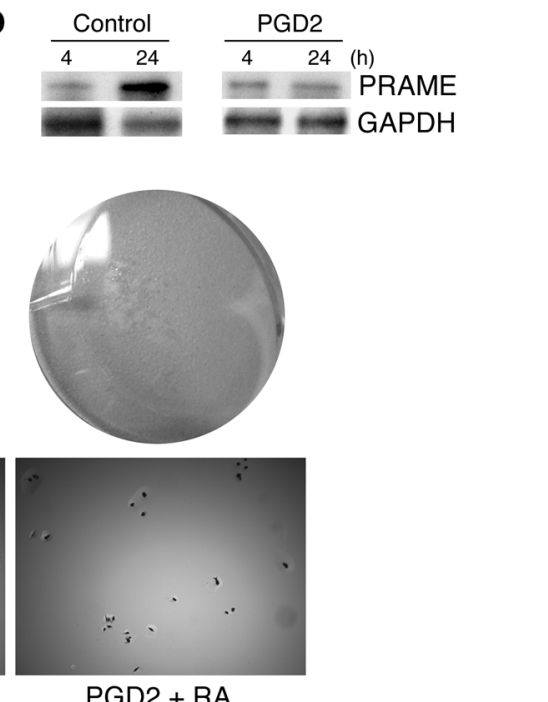

PGD2 + RA

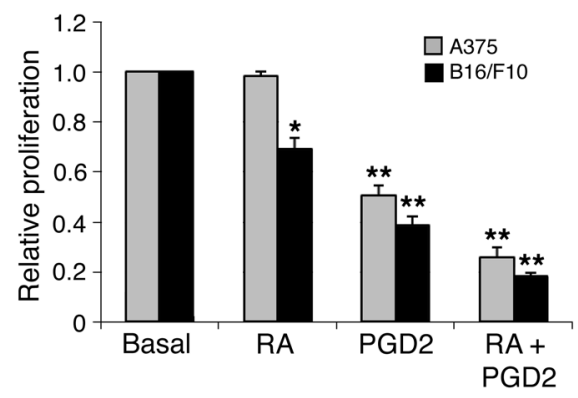

\section{Figure 4}

PGD2 restores RA sensitivity by increasing SOX9 expression, which in turn downregulates PRAME. (A) A375 melanoma cells were transduced with SOX9 lentivirus or with GFP lentivirus as a control. One week after transduction and selection with blasticidin, proteins were extracted and analyzed using immunoblotting with PRAME, SOX9, and GAPDH antibodies (upper panels). Expression levels of PRAME were determined using RT-PCR in A375 cells transfected for 48 hours with GFP or SOX9 cDNA; GAPDH expression served as the control (lower panel). (B) Proliferation assays with $A 375$ and B16/F10 melanoma cells, which are resistant and sensitive to RA, respectively. Addition of RA at $10^{-7} \mathrm{M}$ has no effect on A375 cells but decreases the proliferation of B16/F10 cells. Transfection of SOX9 induces a strong decrease in proliferation in both types of cells. The combination of SOX9 transfection and RA treatment (at 10-7 M) produces an additive decrease in proliferation in both B16/F10 and A375 cells, in which sensitivity to RA was restored. (C) Immunoblotting of A375 cells treated for 48 hours with PGD2 $(0.5 \mu \mathrm{g} / \mu \mathrm{l})$ with or without RA (10 $\left.{ }^{-7} \mathrm{M}\right)$ compared with A375 cells treated in the same conditions but silenced for SOX9 by using an siRNA. Protein expression was analyzed using PRAME, p21, SOX9, and $\beta$-actin antibodies. (D) Metabolic ${ }^{35}$ S labeling of A375 cells treated with $0.5 \mu \mathrm{g} / \mu \mathrm{l}$ PGD2 for 4 and 24 hours. Labeled proteins were immunoprecipitated with PRAME or GAPDH antibodies. (E) Top panels: Proliferation assays of A375 cells treated with RA at $10^{-7}$ M and/or PGD2 at $0.5 \mu \mathrm{g} / \mu \mathrm{l}$ compared with untreated controls. The upper row shows macroscopic views of the proliferative clones and the lower row, the same clones at a magnification of $\times 10$. Bottom panel: Graphic representation of the proliferation assays of A375 and B16/F10 cells treated with RA and/or PGD2. ${ }^{*} P<0.01,{ }^{* *} P<0.001$.

To further study the association of SOX9 and p21 in vivo, we examined the melanoma specimens using immunohistochemistry. Of interest, only the 2 melanomas that showed positive expression of SOX9 in the tissue array (Supplemental Figure 1) also showed a clear expression of $\mathrm{p} 21$, emphasizing the strong relationship between SOX9 and p21 in vivo. It is worth noting that those 2 melanomas were also highly pigmented.
Since SOX9 increases MITF levels in melanoma cells, we first hypothesized that SOX9 may act on p21 by upregulating MITF. However, the relatively small increase in MITF expression after overexpression of SOX9, compared with the marked elevation of p21 that was observed in some melanoma cells such as Mel Juso, suggested that SOX9 might also act directly on the 21 promoter. Analysis of the p21 promoter sequence indicates several potential 
A
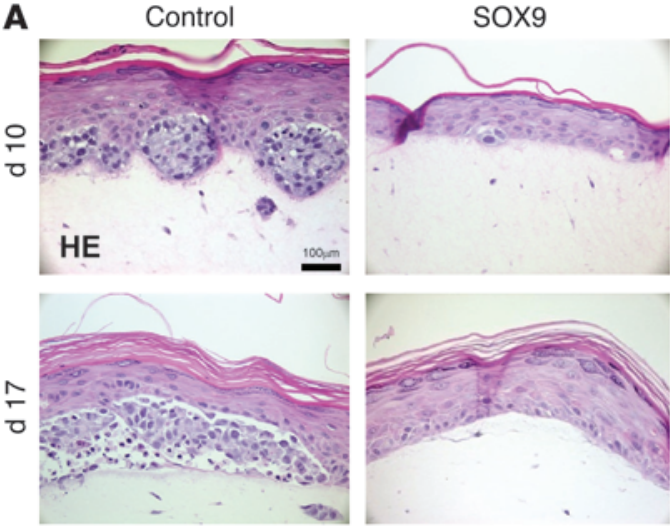

C

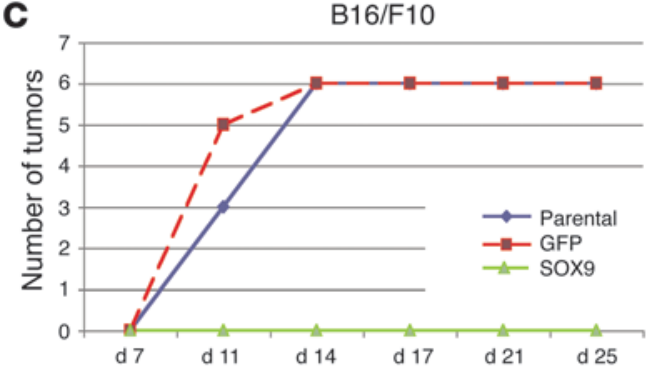

B
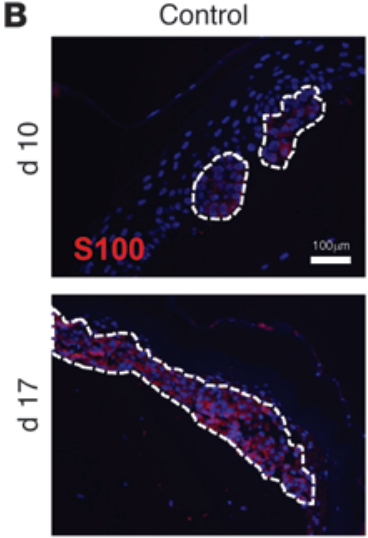
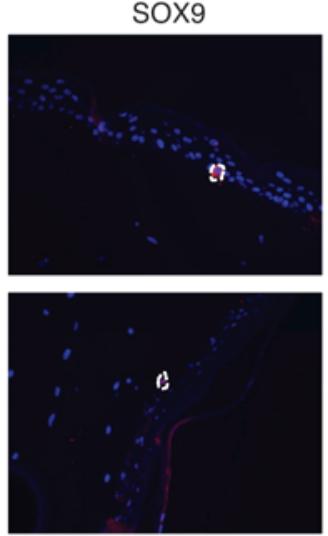

A375

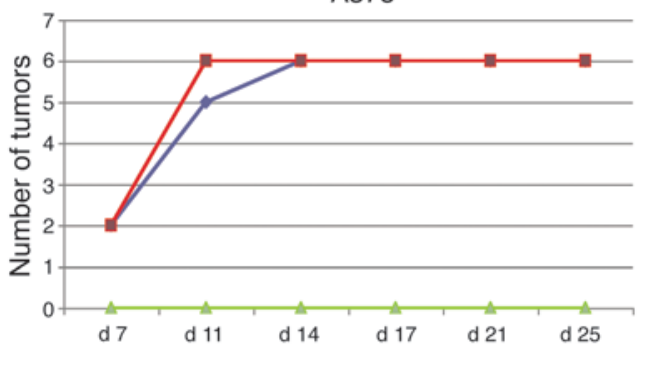

sox9

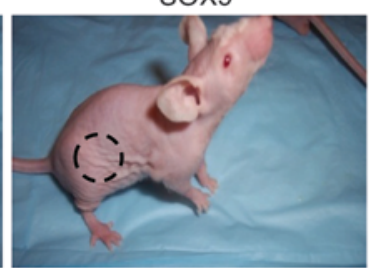

\section{Figure 5}

SOX9 blocks melanoma proliferation in vivo. (A) A375 melanoma cells transduced with SOX9 lentivirus or parental cells as a control were included in a human reconstructed skin model. H\&E staining of melanoma cells shows clear proliferation with some invasiveness of the dermis in the control, while no proliferation was noted in A375 cells transduced with SOX9 at days 10 and 17. (B) Immunostaining of the human reconstructed skin model with $\mathrm{S} 100$ antibody (red) and DAPI (blue). Scale bars: $100 \mu \mathrm{m}$. (C) A375 and B16/F10 melanoma cells, parental (control) or transduced with GFP or SOX9 lentivirus, were injected subcutaneously into nude and C57BL/6 mice, respectively. The graphs show the number of tumors observed in mice from day 7 to day 25 in B16/F10 melanoma-challenged mice (left) and in A375 melanoma-challenged mice (right). The photos of mice show examples of the tumorigenicity of A375 cells in nude mice at day 25 (left to right: A375 parental, A375-GFP, A375SOX9); dashed circles denote injection sites.

consensus DNA binding sequences for SOX9 (12). ChIP assays of A375 melanoma cells, which express both MITF and p21, confirmed that SOX9 binds to the $\mathrm{p} 21$ promoter in vivo under non-stress conditions. No signal above background was seen with nonspecific IgG or with primers specific for the HSP70 promoter as controls (Figure 3E). Thus, the strong increase in $\mathrm{p} 21$ protein expression results both from direct binding of SOX9 to the p21 promoter and from an indirect effect mediated by MITF, which explains the effect of SOX9 on p21 expression in cells in which the induction of MITF by SOX9 is compromised.

SOX 9 restores sensitivity to $R A$ through downregulation of PRAME in melanoma cells. The resistance of cancer cells to RA treatment is due in part to the expression of PRAME (2). We previously demonstrated that SOX9 promotes the differentiation of normal melanocytes and thus sought to determine whether SOX9 would affect the expression of PRAME in melanoma cells. A375 mela- noma cells express high levels of PRAME and are resistant to RA, while B16/F10 cells are sensitive to RA $(2,13,14)$. Immunoblot analysis of A375 cells transfected with SOX9 showed a marked reduction in PRAME protein levels (Figure 4A, upper panel). RT-PCR analysis of A375 cells transfected with SOX9 showed no decrease in PRAME mRNA levels (Figure 4A, lower panel), suggesting a posttranscriptional action of SOX9 on PRAME. To determine whether a reduction in PRAME improves sensitivity to RA treatment, we cultured A375 cells with or without RA for 15 days and then analyzed their proliferative properties. Strikingly, SOX9-expressing cells treated with RA showed a marked decrease in proliferation compared with controls (Figure 4B). In contrast, RA had no effect on the proliferation of untreated cells. Note that in B16/F10 melanoma cells, RA treatment alone decreased proliferation (as expected) but also enhanced the effect of SOX9 transfection. This confirmed that increasing SOX9 expression 
A

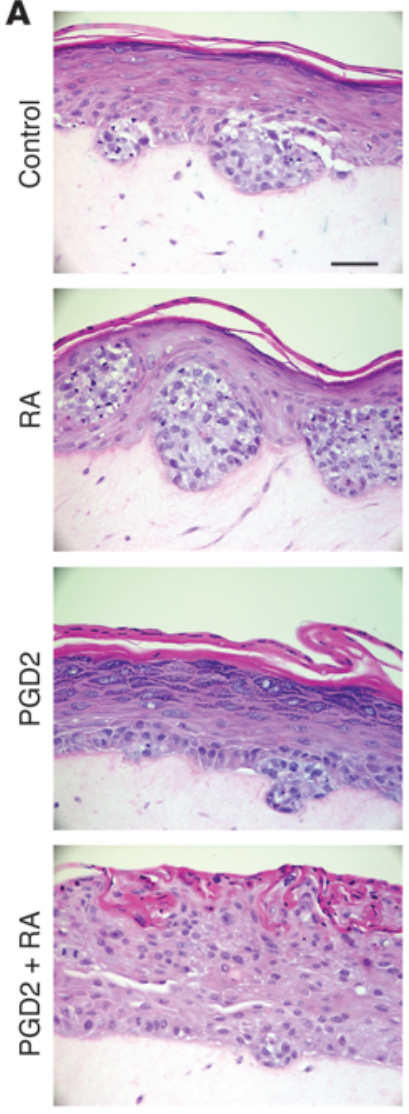

B

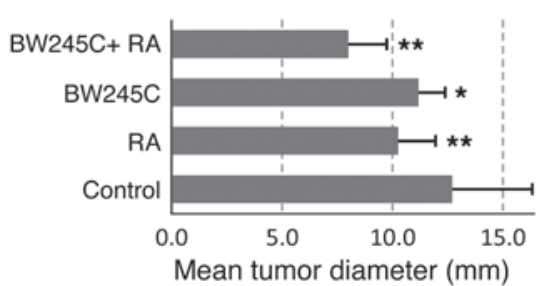

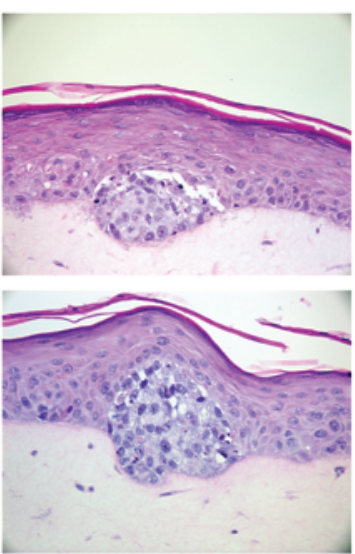
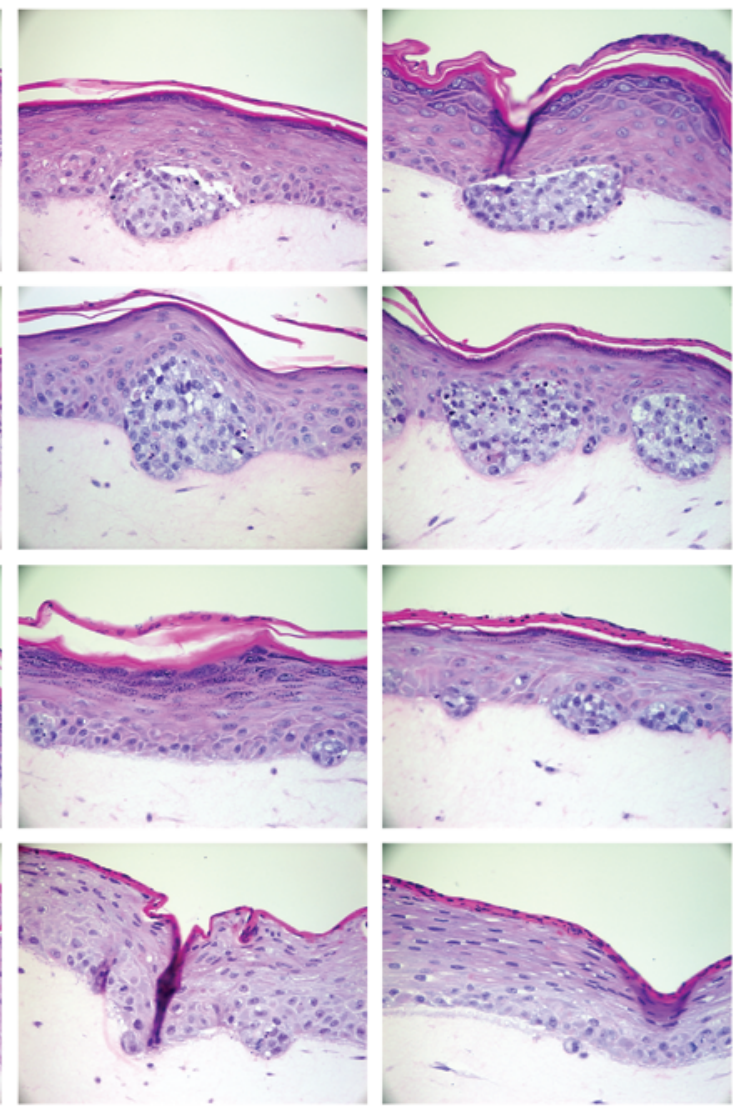

A375

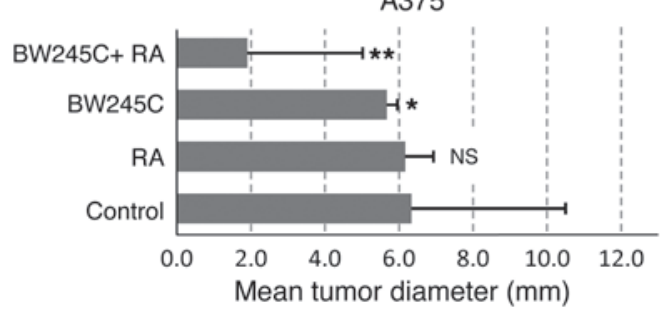

\section{Figure 6}

Combined treatment with PGD2 and RA inhibits melanoma growth in human ex vivo and in mouse models. (A) A375 melanoma cells were included in a human reconstructed skin model and were treated with PGD2 $(0.5 \mu \mathrm{g} / \mu \mathrm{l})$ and/or RA $\left(10^{-7} \mathrm{M}\right)$ for 10 days. H\&E staining of melanoma cells shows a clear proliferation, with some invasiveness of the dermis in the untreated control samples and in RA-treated samples. RA had no effect on tumor growth, but decreased tumor size was observed in melanoma reconstructed skins treated with PGD2, while a marked further decrease in proliferation was noted in samples treated with a combination of PGD2 and RA. Scale bar: $100 \mu \mathrm{m}$. (B) A375 and B16/F10 melanoma cells were injected subcutaneously into nude and C57BL/6 mice, respectively. After 8 days, mice were treated intraperitoneally with BW245C and/or RA 3 times a week for 3 weeks. The graphs show the mean tumor diameters observed after treatment in B16/ F10 melanoma-challenged mice (left) and in A375 melanoma-challenged mice (right). ${ }^{\star} P<0.05,{ }^{*} P<0.01$. restores sensitivity to RA treatment by reducing PRAME expression in melanoma cells.

PGD2 increases endogenous SOX9 expression. The strong antiproliferative effects of SOX9 prompted us to look for agents that may activate SOX9 or upregulate its endogenous expression within melanoma cells. PGD2 and more recently its metabolite $\Delta 2$-PGJ2 have been shown to decrease the proliferation of melanoma and other cancer cells, although their mechanism of action has not been fully elucidated (15-18). It is known that PGD2 increases SOX9 function by promoting its phosphorylation and its nuclear translocation in human testis cancer cells (19). To examine whether SOX9 is regulated by treatment of melanoma cells with PGD2, we grew A375 cells with or without PGD2 for 3 days. Immunoblot analysis confirmed increases in SOX9 and in p21 accompanied by decreased PRAME expression in PGD2-treated cells (Figure 4C, left panels, and Supplemental Figure 2). For further confirmation of these results, cells were treated with or without PGD2, then were metabolically labeled with ${ }^{35} \mathrm{~S}$ and chased for 4 hours and 24 hours. In untreated cells, PRAME levels increased after the 24-hour chase. However, PGD2 kept PRAME levels low after the same period of time (Figure 4D).

Additive action of SOX9 and RA in treatment of melanoma. Our observations thus suggest a novel regulatory mechanism whereby SOX 9 downregulates PRAME, restoring sensitivity to RA in melanoma cells resistant to that therapy. Therefore, we hypothesized that combining treatment with PGD2 and RA would inhibit the proliferation of melanoma cells usually resistant to RA. To test this, A375 cells were left untreated or were treated with RA and/or PGD2 for 15 days. Proliferation assays showed a 50\% decrease in proliferation in cells treated with PGD2 alone compared with untreated cells, whereas RA alone had no effect on proliferation (Figure 4E). Strikingly, the combination of PGD2 and RA further reduced proliferation by $75 \%$, suggesting that PGD2 restores, at least in part, the sensitivity of A375 cells to RA. For B16/F10 melanoma cells, RA or PGD2 treatment reduced proliferation, while RA combined with PGD2 led to an additive decrease in proliferation. Immunoblot assays performed on B16/F10 cells treated with RA showed an increase in SOX9 expression, whereas no effect 
on SOX9 was observed in A375 cells treated in the same conditions (Supplemental Figure 3).

To further elucidate the role of SOX9 in mediating the effects of RA and PGD2 treatments on PRAME and p21 expression, we used a specific SOX9 siRNA probe on A375 melanoma cells. Immunoblot analysis confirmed that the combination of PGD2 and RA induced greater expression of SOX9 protein compared with PGD2 treatment alone (Figure 4C). The increase in p21 expression and the decrease in PRAME expression were also enhanced when PGD2 was combined with RA. In comparison, when SOX9 was silenced, PGD2 in the presence or absence of RA had no effect on p21 or PRAME expression.

Overexpression of SOX9 prevents the proliferation of melanoma cells in buman melanoma reconstructed skin and in mouse melanoma models. The strong inhibition of proliferation observed in melanoma cells in vitro following overexpression of SOX9 led us to test the tumorigenic potential of those cells in vivo. First, we used a melanoma reconstructed skin model in which melanocytes were replaced by A375 melanoma cells with or without stable transfection with SOX9. After 10 days, several tumors were observed within the epidermis, and evidence of early metastasis into dermal structures was observed with A375 control cells (Figure 5A). In contrast, no tumor formation was observed with A375-SOX9 cells. Similar patterns were also observed at day 17. All tumor cells showed expression of the S100 protein, a known melanoma marker (Figure 5B). A375-SOX9 melanoma reconstructed skins showed moderate but clear staining of SOX9 in the nuclei of melanoma cells, whereas SOX9 staining in A375-control cells was negative, except for some cells (circled), where staining was noted in the cytoplasm. Interestingly, costaining for MITF corroborated the in vitro data, showing clear expression of MITF in A375-SOX9 cells and none in the A375 controls (Supplemental Figure 4).

In an independent approach, A375 and B16 cells were transduced with GFP or SOX9 lentivirus and, after 7 days of selection, SOX9 expression was analyzed by immunoblotting (data not shown). A375 and B16 cells were then injected subcutaneously into nude and C57BL/6 mice, respectively. By day 25, both parental and GFPtransduced melanoma cells induced tumors in all mice, whereas SOX9-transduced cells did not induce any tumors (Figure 5C).

Activation of the PGD2 pathway combined with RA provides useful treatment of melanoma tumors in human and mouse melanoma models. Melanoma reconstructed skins with parental A375 melanoma cells were treated with PGD2 and/or RA. After 10 days, several tumors were observed within the epidermis, and evidence of early metastasis into dermal structures was observed in untreated skins and also in skins treated with RA (Figure 6A). However, tumor growth was significantly reduced after PGD2 treatment, and a marked further decrease in proliferation was observed when PGD2 and RA treatment were combined.

BW245C is a PGD2 agonist that has been used in humans to test platelet aggregation responses and intraocular pressure (20-22). We injected A375 and B16/F10 melanoma cells subcutaneously in nude and C57BL/ 6 mice, respectively. After 8 days, subcutaneous tumors formed, and treatment with BW245C and/or RA was given intraperitoneally 3 times a week for 3 weeks. At the end of the treatment regimen, BW245C-treated nude and C57BL/6 mice showed slight but significant decreases in tumor size compared with untreated controls (Figure 6B). RA treatment alone decreased B16 tumor size but had no effect on A375 tumors. In B16 and A375 tumors, the combination of BW245C and RA induced marked and significant decreases in tumor size compared with controls. Immu- nohistochemistry performed on those tumors showed increases in SOX9 and MITF expression in BW245C- and in BW245C plus RA-treated tumors (Supplemental Figure 5).

\section{Discussion}

In this study, we demonstrate a critical role for SOX9 in the regulation of melanoma cell proliferation and suggest a novel approach to targeting malignant melanoma by upregulating SOX9 expression. SOX9 has been increasingly reported to be expressed in various adult tissues (5-7), and we recently reported its expression in normal human melanocytes in the skin and its role in UVB-induced melanogenesis (8). A potential role of SOX9 has also been reported in breast, prostate, ovarian, and colon cancers (10, 23-25). Here, we shed light on the mechanisms by which SOX9 decreases proliferation and promotes the differentiation of malignant cells. We demonstrate that increased expression of SOX9 effectively inhibits the proliferation of melanoma cells and leads to complete abolition of tumorigenicity in mouse and in human ex vivo skin models. This effect is achieved by 2 distinct mechanisms: (a) an indirect effect, whereby SOX9 activates MITF, which in turn acts on p21 and blocks melanoma proliferation $(9,11)$; and (b) a direct effect, whereby SOX 9 directly binds the p 21 promoter and increases its expression. Interestingly, the increase in MITF was found to be more dramatic in A375 and B16/F10 cells than in Mel Juso melanoma cells, suggesting that alternative mechanisms might regulate SOX9's effects on MITF and/or p21.

To the best of our knowledge, no protein has been previously reported that downregulates the expression of PRAME. Further, overexpression of SOX9, most probably through its action on PRAME, restores the sensitivity of melanoma cells to RA, which leads to an additive inhibitory effect on proliferation. Our data show that PRAME transcription is not affected by the overexpression of SOX9, which suggests a posttranscriptional regulatory mechanism. The mechanism of action of SOX9 on PRAME expression warrants further study. This downregulation of PRAME opens encouraging therapeutic perspectives not only for melanoma but also potentially for all cancers that are resistant to RA due to the expression of PRAME. Interestingly, RA has also been shown to increase SOX9 expression in a cartilage-derived cell line (26), and RA receptor agonists stimulate SOX9 gene expression in some breast cancer cell lines (10). We also found increased expression of SOX9 after RA treatment of B16 melanoma cells (which are naturally sensitive to RA) but no increase in SOX9 in A375 cells. By restoring RA sensitivity in melanoma cells, the RA treatment also leads to a secondary increase in SOX9, which further potentiates its effects. Thus, we envision that genetic therapy using an adenovirus or a lentivirus expressing SOX9 controlled by a cell type-specific promoter could lead to strong and specific effects on the growth and metastasis of melanomas or other cancers, especially if combined with RA treatment.

The identification of molecules that could increase or activate SOX9 expression without requiring genetic therapy would be extremely useful. SOX9 has already been widely studied for its role in chondrogenesis, and several agents have been reported to increase its activity. For example, calcineurin inhibitors are able to increase the phosphorylation of SOX9 and thus its translocation to the nucleus (27). Interestingly, a new calcineurin inhibitor without immunomodulatory effects has shown promising results in treating melanomas (28). Deacetylase inhibitors can increase SOX9 expression not only in chondrocytes but also in sarcomas $(29,30)$. Here again, recent reports showed the usefulness of such 
agents in treating various types of cancers, including melanomas (31). Some of the most interesting agents appear to be PGD2 and its derivatives. PGD2 increases the expression and phosphorylation of SOX9 in human testicular carcinoma NT2/D1 cells by specifically activating its DP1 receptor (19). PGD2 and its metabolite $\Delta 2$-PGJ2 were shown to decrease the proliferation of melanomas and other cancers, although their mechanism of action was not fully elucidated (15-18). Indeed, PGD2 induces cell cycle arrest in those cancers and increases p 21 expression, but the pathway involved remains unknown. In this study, we show that PGD2 increases the expression of SOX9 in melanoma cells and also leads to the decreased expression of PRAME. Moreover, the increased expression of $\mathrm{p} 21$ and decreased expression of PRAME induced by PGD2 treatment were prevented by the silencing of SOX9, showing the key role of SOX9 in the effects of PGD2 on melanoma cells.

The antiproliferative effect of SOX9 and its activation through the PGD2 pathway (via a DP1 agonist) have been recently described in ovarian cancer cells (25). Although that study did not examine the mechanism of action of SOX9, it corroborates our findings and strongly suggests that activating SOX9 through the PGD2 pathway might be a useful approach to treat not only melanomas but also other solid tumors. We were able to show the additive effect of PGD2 and RA, even in melanoma cell lines usually resistant to RA. These effects were confirmed in human ex vivo models and in mice, where treatment with PGD2 or a DP1 agonist combined with RA led to a significant decrease in tumor size in both RA-resistant and RA-sensitive cells. The doses and the rates of administration of those products still have to be optimized, but the PGD2-dependent sensitization to RA suggests useful noncytotoxic therapeutic approaches. Additionally, SOX9 is an attractive target because it is a growth suppressor factor that does not appear to be completely suppressed in melanomas. In fact, treatment with PGD2 restored expression of the endogenous SOX9 gene. Finally, SOX9 was downregulated in more than $96 \%$ of primary melanoma specimens and in $100 \%$ of metastatic melanoma specimens tested and is therefore presumably a potential target for the vast majority of melanomas.

Thus, the findings that SOX9 overexpression downregulates melanoma cell proliferation (through direct and indirect stimulation of the $\mathrm{p} 21$ promoter) and restores sensitivity to RA by acting on PRAME provide new insight into our understanding of melanoma cell pathophysiology. Importantly, they suggest potential combination therapies to treat melanomas and other RA-resistant cancers. Further screening and identification of agents effective in increasing or activating SOX9, such as PGD2 metabolites or specific agonists of the DP1 receptor, and combining them with RA therapy should provide a powerful approach to melanoma therapy.

\section{Methods}

Cell culture and reagents. B16/F10, SK-Mel 28, and A375 melanoma cells were obtained from ATCC. Mel Juso cells were a gift from Thomas Hornyak at the National Cancer Institute (Bethesda, Maryland, USA). A375, SK-Mel 28, and $\mathrm{B} 16$ cells were grown at $37^{\circ} \mathrm{C}$ under $5 \% \mathrm{CO}_{2}$ in DMEM supplemented with $7 \% \mathrm{FBS}$, penicillin $(100 \mathrm{U} / \mathrm{ml})$, and streptomycin $(50 \mu \mathrm{g} / \mathrm{ml})$. Mel Juso cells were grown at $37^{\circ} \mathrm{C}$ under $5 \% \mathrm{CO}_{2}$ in RPMI medium supplemented with $10 \% \mathrm{FBS}$, penicillin $(100 \mathrm{U} / \mathrm{ml})$, and streptomycin $(50 \mu \mathrm{g} / \mathrm{ml})$. RA, PGD2, and BW245C were purchased from Sigma-Aldrich.

Reconstructed skin. The melanoma skin model was obtained from MatTek Corp. Normal human keratinocytes and fibroblasts were obtained from neonatal foreskin tissues of individuals of European descent (MatTek Corp.). A375 melanoma cells (parental or transduced with SOX9 lentivirus) were added to the skin constructs. The melanoma skin models were grown at the air/liquid interface with the maintenance MCDB 153 Basal Medium (MatTek Corp.) underneath. The culture medium was replenished every 2 days. Experiments were performed in duplicate and were repeated at least once independently. When PGD2 and RA were used, they were added directly into the medium.

Plasmid and lentivirus construction, siRNA, and transfection. The SOX9 cDNA was purchased from the ATCC and after amplification was ligated into the pcDNA3.1 vector (Invitrogen). The constructs were confirmed by sequence analysis. As a negative control, the pcDNA3.1 vector with no insertion was used. Transfection was performed for melanocytes using Lipofectamine 2000 (Invitrogen). Cells were seeded 1 day in advance and were transfected according to the manufacturer's instructions. ON-TARGETplus SMARTpool (Dharmacon, Thermo Scientific) siRNA for human p21 and human SOX9 was used to silence 21 and SOX9 expression, respectively. A commercial negative control sequence (Invitrogen) was used to monitor for off-target effects.

Transfections for silencing experiments were performed using RNAiMAX (Invitrogen). The amount of DNA used for each transfection was $2 \mu \mathrm{g}$ per $10^{6}$ cells, whereas siRNA was used at $100 \mathrm{nM}$. The SOX 9 and GFP lentivirus were generated by cloning the SOX9 or GFP cDNA into the pLenti 6.2 (Invitrogen).

Immunoblotting, immunohistochemistry, and immunocytochemical staining. Full details are provided in Supplemental Methods.

Proliferation assay. Cells were transfected with a pBabe vector to give them resistance to puromycin. At the same time, cells were transfected with SOX9 cDNA or an empty vector as a control. Depending on the experiment, cells were also transfected the day after with siRNA p21 or without siRNA (mock). After 10-15 days, the clones were fixed and stained with crystal violet. Proliferation was measured as optical density.

FACS analysis. Cells were harvested and incubated at $4{ }^{\circ} \mathrm{C}$ for 1 hour with a solution containing $0.1 \%$ Triton X-100, $0.1 \% \mathrm{Na}$ citrate, and $50 \mu \mathrm{g} / \mathrm{ml}$ propidium iodide. Cell cycle analysis was performed using a FACSCalibur (BD) and CellQuest Pro software (BD).

Metabolic labeling. Full details are provided in Supplemental Methods.

Chromatin immunoprecipitation. Full details are provided in Supplemental Methods.

Animal model. All animal experiments were approved by the NCI-Bethesda Animal Care and Use Committee of the National Cancer Institute. One week after transduction and selection, $1 \times 10^{6} \mathrm{~B} 16$ mouse melanoma cells (parental, GFP-transduced, or SOX9-transduced) were injected subcutaneously into C57BL/ 6 mice, while $10^{6}$ human melanoma A375 melanoma cells (parental, GFP-transduced, or SOX9-transduced) were injected into nude mice. Mice were then monitored for date of tumor appearance and tumor size until day 25 or until the tumor measured $2 \mathrm{~cm}$ in diameter. The second set of mouse experiments was performed with $1 \times 10^{6} \mathrm{~B} 16$ mouse melanoma cells and with $5 \times 10^{6}$ human melanoma A375 melanoma cells injected subcutaneously into C57BL/6 and nude mice, respectively. After 8 days, mice were treated intraperitoneally with RA ( $2 \mathrm{mg} / \mathrm{mouse} / \mathrm{d})$ and/or BW245C ( $3 \mu \mathrm{g} / \mathrm{mouse} / \mathrm{d}) 3$ times a week for 3 weeks. All experiments were done in 18 mice ( 6 mice/group) in duplicate in 2 separate experiments.

Statistics. Data are presented as mean \pm SD. Two-tailed Student's $t$ test was used to analyze differences. $P$ values less than 0.05 were considered significant.

Note added in proof. Since this article was accepted for publication, the authors have tested the staining of SOX9 in the nevi and melanoma arrays under the same conditions as shown in Figure 1 but 
using an antibody from a different commercial source (Chemicon, catalog no. AB5535). The staining patterns were consistent with the patterns shown in Figure 1 (in which the SOX9 antibody from Abcam, catalog no. ab3697, was used), although the localization was more nuclear and was more restricted to the melanocytic cells in the tissues. There continued to be decreased expression of SOX9 during melanocyte progression to melanoma, but reactivity was higher, and more cells appeared positive (100\% of nevi, $90 \%$ of primary melanomas, and $\sim 75 \%$ of metastatic melanomas).

\section{Acknowledgments}

This research was supported by the Intramural Research Program of the NIH, National Cancer Institute. We thank Sergio C. Coelho for providing sections of human skin and Michael M.
Gottesman and Douglas M. Lowy for helpful discussions about the study and manuscript.

Received for publication December 16, 2008, and accepted in revised form January 14, 2009.

Address correspondence to: Vincent J. Hearing, Laboratory of Cell Biology, National Cancer Institute, National Institutes of Health, Building 37, Room 2132, MSC 4256, Bethesda, Maryland 20892, USA. Phone: (301) 496-1564; Fax: (301) 402-8787; E-mail: hearingv@nih.gov.

Thierry Passeron's present address is: Department of Dermatology, University Hospital of Nice, Nice, France.
1. Ikeda, H., et al. 1997. Characterization of an antigen that is recognized on a melanoma showing partial HLA loss by CTL expressing an NK inhibitory receptor. Immunity. 6:199-208.

2. Epping, M.T., et al. 2005. The human tumor antigen PRAME is a dominant repressor of retinoic acid receptor signaling. Cell. 122:835-847.

3. Foster, J.W., et al. 1994. Campomelic dysplasia and autosomal sex reversal caused by mutations in an SRY-related gene. Nature. 372:525-530.

4. Kwok, C., et al. 1995. Mutations in SOX9, the gene responsible for Campomelic dysplasia and autosomal sex reversal. Am. J. Hum. Genet. 57:1028-1036.

5. Akiyama, H., et al. 2004. Essential role of Sox9 in the pathway that controls formation of cardiac valves and septa. Proc. Natl. Acad. Sci. U. S. A. 101:6502-6507.

6. Pepicelli, C.V., Kispert, A., Rowitch, D.H., and McMahon, A.P. 1997. GDNF induces branching and increased cell proliferation in the ureter of the mouse. Dev. Biol. 192:193-198.

7. Pompolo, S., and Harley, V.R. 2001. Localisation of the SRY-related HMG box protein, SOX9, in rodent brain. Brain Res. 906:143-148.

8. Passeron, T., et al. 2007. SOX9 is a key player in ultraviolet B-induced melanocyte differentiation and pigmentation. Proc. Natl. Acad. Sci. U. S. A. 104:13984-13989.

9. Carreira, S., et al. 2005. Mitf cooperates with Rb1 and activates p21Cip1 expression to regulate cell cycle progression. Nature. 433:764-769.

10. Afonja, O., et al. 2002. RAR agonists stimulate SOX9 gene expression in breast cancer cell lines: evidence for a role in retinoid-mediated growth inhibition. Oncogene. 21:7850-7860.

11. Wellbrock, C., and Marais, R. 2005. Elevated expression of MITF counteracts B-RAF-stimulated melanocyte and melanoma cell proliferation. J. Cell Biol. 170:703-708.

12. Panda, D.K., Miao, D., Lefebvre, V., Hendy, G.N., and
Goltzman, D. 2001. The transcription factor SOX9 regulates cell cycle and differentiation genes in chondrocytic CFK2 cells. J. Biol. Chem. 276:41229-41236.

13. Gruber, J.R., Desai, S., Blusztajn, J.K., and Niles, R.M. 1995. Retinoic acid specifically increases PKC $\alpha$ and stimulates AP-1 transcriptional activity in B16 mouse melanoma cells. Exp. Cell Res. 221:377-384.

14. Lotan, R., and Nicolson, G.L. 1979. Heterogeneity in growth inhibition by $\beta$-trans-retinoic acid of metastatic B16 melanoma clones and in vivo-selected cell variant lines. Cancer Res. 39:4767-4771.

15. Fitzpatrick, F.A., and Stringfellow, D.A. 1979. Prostaglandin D2 formation by malignant melanoma cells correlates inversely with cellular metastatic potential. Proc. Natl. Acad. Sci.U. S. A. 76:1765-1769.

16. Stringfellow, D.A., and Fitzpatrick, F.A. 1979. Prostaglandin D2 controls pulmonary metastasis of malignant melanoma cells. Nature. 282:76-78.

17. Bhuyan, B.K., Adams, E.G., Badiner, G.J., Li, L.H., and Barden, K. 1986. Cell cycle effects of prostaglandins A1, A2, and D2 in human and murine melanoma cells in culture. Cancer Res. 46:1688-1693.

18. McClay, E.F., Winski, P.J., Jones, J.A., Jennerette, J., 3rd, and Gattoni-Celli, S. 1996. delta 12-Prostaglandin-J2 is cytotoxic in human malignancies and synergizes with both cisplatin and radiation. Cancer Res. 56:3866-3869.

19. Malki, S., et al. 2005. Prostaglandin D2 induces nuclear import of the sex-determining factor SOX9 via its CAMP-PKA phosphorylation. EMBOJ. 24:1798-1809.

20. Al-Sinawi, L.A., et al. 1985. Effect of a hydantoin prostaglandin analogue, BW245C, during oral dosing in man. Prostaglandins. 29:99-111.

21. Nakajima, M., Goh, Y., Azuma, I., and Hayaishi, O. 1991. Effects of prostaglandin D2 and its analogue, BW245C, on intraocular pressure in humans. Graefes Arch. Clin. Exp. Ophthalmol. 229:411-413.

22. Shah, A., Pickles, H., Joshi, M., Webster, A., and O'Grady, J. 1984. Effects of single oral dose admin- istration of a hydantoin prostaglandin analogue BW 245C in man. Life Sci. 34:2281-2286.

23. Drivdahl, R., et al. 2004. Suppression of growth and tumorigenicity in the prostate tumor cell line M12 by overexpression of the transcription factor SOX9. Oncogene. 23:4584-4593.

24. Jay, P., Berta, P., and Blache, P. 2005. Expression of the carcinoembryonic antigen gene is inhibited by SOX9 in human colon carcinoma cells. Cancer Res. 65:2193-2198.

25. Malki, S., et al. 2007. Expression and biological role of the prostaglandin D synthase/SOX9 pathway in human ovarian cancer cells. Cancer Lett. 255:182-193.

26. Sekiya, I., et al. 2000. SOX9 enhances aggrecan gene promoter/enhancer activity and is up-regulated by retinoic acid in a cartilage-derived cell line, TC6. J. Biol. Chem. 275:10738-10744.

27. Zakany, R., et al. 2005. Hydrogen peroxide inhibits formation of cartilage in chicken micromass cultures and decreases the activity of calcineurin: implication of ERK1/2 and Sox9 pathways. Exp. Cell Res. 305:190-199.

28. Ciechomska, I., et al. 2005. Cyclosporine A and its non-immunosuppressive derivative NIM811 induce apoptosis of malignant melanoma cells in in vitro and in vivo studies. Int. J. Cancer. 117:59-67.

29. Furumatsu, T., et al. 2005. Sox 9 and p 300 cooperatively regulate chromatin-mediated transcription. J. Biol. Chem. 280:35203-35208.

30. Liu, S., Cheng, H., Kwan, W., Lubieniecka, J.M., and Nielsen, T.O. 2008. Histone deacetylase inhibitors induce growth arrest, apoptosis, and differentiation in clear cell sarcoma models. Mol. Cancer Ther. 7:1751-1761.

31. Peltonen, K., Kiviharju, T.M., Jarvinen, P.M., Ra, R., and Laiho, M. 2005. Melanoma cell lines are susceptible to histone deacetylase inhibitor TSA provoked cell cycle arrest and apoptosis. Pigment Cell Res. 18:196-202. 